\title{
Are the garment seams a potential source of breach in level-4 personal protective equipment gowns?
}

\author{
Evan Wild, MBBS, BMedSci, FRCA, FRCPC • Fabricio B. Zasso, MD • \\ Kong Eric You-Ten, MD, PhD, FRCPC
}

Received: 2 December 2020/Revised: 14 December 2020/Accepted: 22 December 2020/Published online: 23 February 2021

(C) Canadian Anesthesiologists' Society 2021

\section{To the Editor,}

Personal protective equipment (PPE) is essential for reducing potentially life-threatening infections in healthcare workers, as highlighted by the recent transmission of severe acute respiratory syndrome coronavirus 2. ${ }^{1}$ The Association for the Advancement of Medical Instrumentation has classified surgical gowns into four categories based on the level of protection they offer, with level 1 protecting against only water spray contact, and level 4 protecting against blood and virus penetration under pressure for limited time periods. ${ }^{2}$ The recognition of wearing higher protection level gowns (level 4) during high-risk situations, such as aerosol-generating medical procedures, has recently been highlighted. ${ }^{3}$ Shortages of single-use high-level PPE have been well reported, ${ }^{4}$ with many institutions opting for various reusable PPE gowns of various protection levels. ${ }^{5}$

To inform our strategy for using higher protection level gowns in high-risk situations, we simulated the spread of cough-generated particles to investigate the degree to which aerosols can penetrate gowns of various protective levels.

We prepared an aerosolizing contrast medium consisting of the ink core of two yellow highlighter pens (Sharpie; Atlanta, GA, USA) that were left soaking for two hours in $250 \mathrm{~mL}$ water. This medium was then placed into an aerosol-generating device (Eyeglass Lens Spray Bottle Bloor Optical, Toronto, ON, Canada). The outer surface of each gown's body was sprayed twice with the medium

E. Wild, MBBS, BMedSci, FRCA, FRCPC .

F. B. Zasso, MD - K. E. You-Ten, MD, PhD, FRCPC ( $ه)$.

Department of Anesthesia and Pain Medicine, Mount Sinai

Hospital, University of Toronto, Toronto, ON, Canada

e-mail: eric.you-ten@sinaihealth.ca from $30 \mathrm{~cm}$. A single investigator ran a finger on the inner surface of the gown with clean nitrile gloves (Pri-Med, Edmonton, $\mathrm{AB}$, Canada) to assess whether any of the aerosolized medium had penetrated to the gown interior. A blacklight flashlight (Vanksy 51Led UV Flashlight, Vanskytech ${ }^{\circledR}$, Shenzen, China) was used to visualize the aerosolized contrast medium "glowing" on the gloves to determine contamination of the gown's interior. We assessed four types of waterproof gown used in our institution: 1) level-2 reusable gowns (K-Bro Linen Systems, Toronto, ON, Canada) $(n=3), 2)$ level-3 disposable gowns by MedPro Defense (Model 018-300, Cardinal Health Canada, Vaughan, ON, Canada) $(n=3), 3)$ level-4 reusable gown with breathable Mortex material by Lac-Mac (Model 5XEAC, Lac-Mac, London, ON, Canada) $(n=4)$, and 4) level-4 disposable gowns with nonbreathable polyethylene material by Sirus (Model DYNJP2228S, Medline, Mundelein, IL, USA) $(n=3)$.

We observed no penetration of the contrast fluid to the gowns' inner surfaces when the aerosolized medium made contact with the outer main body. In all level-2 gowns ( $n=$ $3 / 3$, K-Bro Linen), two level-4 reusable gowns $(n=2 / 4$, Lac-Mac, Figure, panels A-C), and one level-4 disposable gown $(n=1 / 3$, Sirus, Figure, panels D-F), we observed transfer of the contrast fluid on a gloved finger pressed along the interior seams, suggesting contamination along the seam lines. Contamination of the interior seam lines was not observed immediately, but occurred ten minutes after exposure to the aerosolized contrast medium.

Based on our findings, we continue to advocate wearing higher-level gowns in high-risk situations. Nevertheless, our findings raise the question of whether the seam line of the main body of gowns can potentially serve as a structural route through which contaminated aerosols generated during high-risk procedures penetrate and 


\section{Lac-Mac}
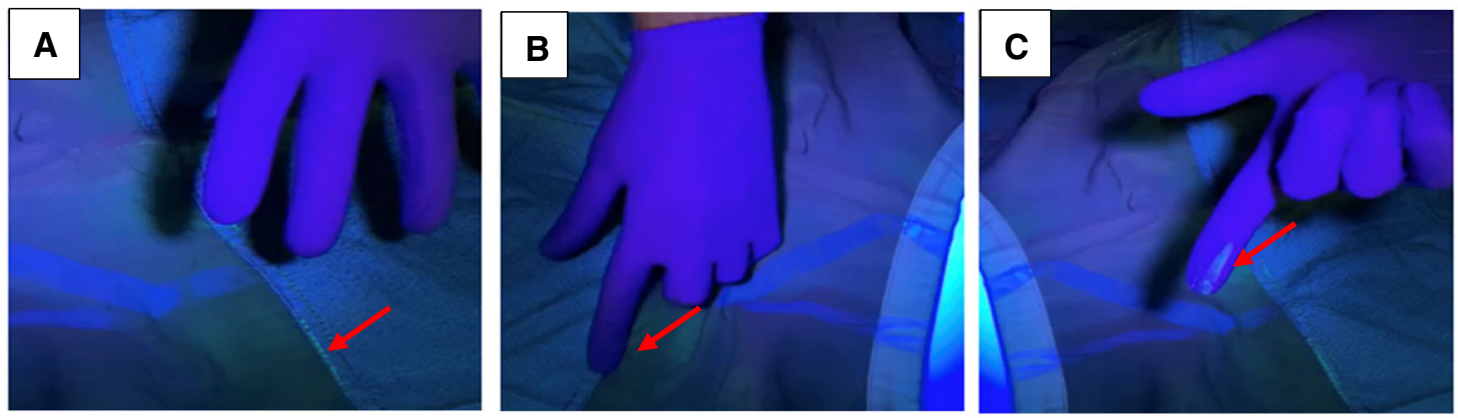

\section{Sirus}
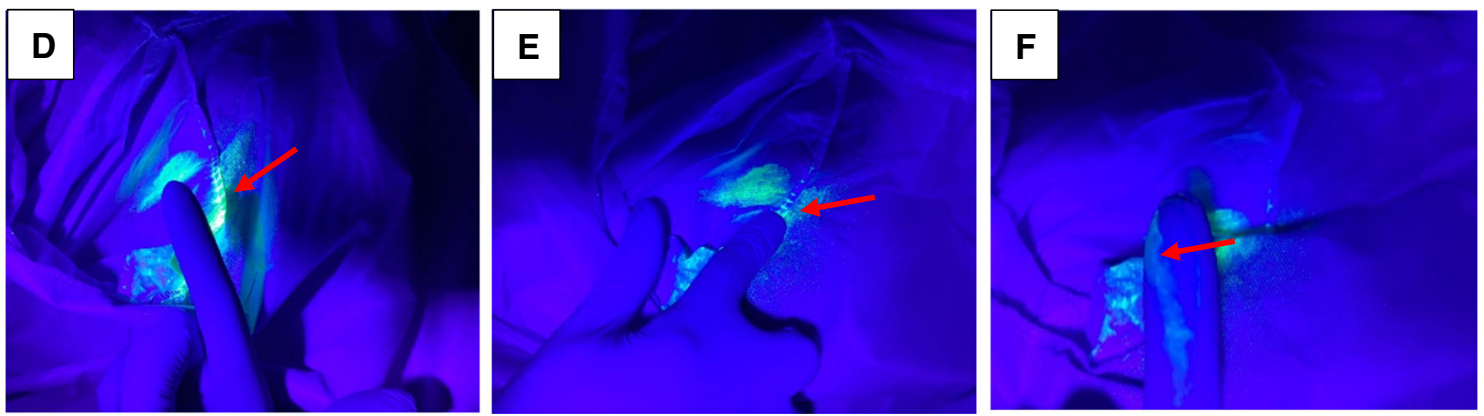

Figure Interior of the level-4 reusable Lac-Mac (Model 5XEAC, Lac-Mac, London, ON, Canada) and the disposable Sirus (Model DYNJP2228S, Medline, Mundelein, IL, USA) protective gowns along the seam lines following contact with a glow medium. Luminescence can be seen along the seam line of the interior of the gown (Figure, panels A, D; arrow) and absence of glove contamination prior to contact with the gown. Upon rubbing a single finger along the interior seam (B, E; arrow), gross contamination (C,F; arrow) of the practitioner's glove is shown.

contaminate the interior of procedural surgical gowns. We recognize limitations in our study. Firstly, this was a qualitative and not quantitative study. Further instruments to measure droplet size and count would be needed for quantitative analysis. Furthermore, our study investigated only gross macroscopic but not microscopic contamination. Our method of aerosolization remains limited in that it may not accurately represent a cough in terms of droplet size or aerosolizing potential.

Disclosures None.

\section{Funding statement None.}

Editorial responsibility This submission was handled by Dr. Hilary P. Grocott, Former Editor-in-Chief, Canadian Journal of Anesthesia.

\section{References}

1. Meng $L$, Qiu H, Wan $L$, et al. Intubation and ventilation amid the COVID-19 outbreak: Wuhan's Experience. Anesthesiology 2020; 132: 1317-32.
2. U.S. Food and Drug Administration. March 11th 2020; Medical Gowns. Available from URL: https://www.fda.gov/medicaldevices/personal-protective-equipment-infection-control/medicalgowns\#g. Accessed December 2020.

3. Lockhart SL, Duggan LV, Wax RS, Saad S, Grocott HP. Personal protective equipment (PPE) for both anesthesiologists and other airway managers: principles and practice during the COVID-19 pandemic. Can J Anesth 2020. DOI:https://doi.org/10.1007/ s12630-020-01673-w.

4. World Health Organisation. Shortage of personal protective equipment endangering health workers worldwide. Available from URL: https://www.who.int/news-room/detail/03-03-2020shortage-of-personal-protective-equipment-endangering-healthworkers-worldwide. Accessed December 2020.

5. Centres for Disease Control and Prevention. Strategies for Optimizing the Supply of Isolation Gowns. Available from URL: https://www.cdc.gov/coronavirus/2019-ncov/hcp/ppe-strategy/ isolation-gowns.html. Accessed December 2020.

Publisher's Note Springer Nature remains neutral with regard to jurisdictional claims in published maps and institutional affiliations. 